\title{
Tilt plane orientation in antiferroelectric liquid crystal cells and the origin of the pretransitional effect
}

\author{
P. Rudquist, J. P. F. Lagerwall, J. G. Meier, K. D’havé, and S. T. Lagerwall \\ Department of Microelectronics and Nanoscience, School of Physics and Engineering Physics, Chalmers University of Technology, \\ S-412 96 Göteborg, Sweden
}

(Received 14 May 2002; published 30 December 2002)

\begin{abstract}
The optic, electro-optic, and dielectric properties of antiferroelectric liquid crystals (AFLCs) are analyzed and discussed in terms of the local tilt plane orientation. We show that the so-called pretransitional effect is a combination of two different electro-optic modes: the field-induced antiphase distortion of the antiferroelectric structure and the field-induced reorientation of the tilt plane. In the presence of a helix, the latter corresponds to a field-induced distortion of the helix. Both electro-optic modes are active only when the electric field has a component along the tilt plane. Thus, by assuring a horizontal surface-stabilized condition, where the helix is unwound by surface action and the tilt plane is everywhere parallel to the cell plates, the pretransitional effect should be suppressed. We also discuss the dielectrically active modes in AFLCs and under which circumstances they contribute to the measured dielectric permittivity.
\end{abstract}

DOI: 10.1103/PhysRevE.66.061708

PACS number(s): 61.30.-v, 77.84.Nh, 61.30.Gd, 77.84.-s

\section{INTRODUCTION}

Antiferroelectric liquid crystals (AFLCs) [1] are a subclass of tilted smectics in which the director $\mathbf{n}$ in adjacent layers tilts in opposite directions $\pm \theta$ with respect to the layer normal $\mathbf{z}$. The most important antiferroelectric liquid crystal phase is the smectic $C_{a}^{*}$ phase, where " $C$ " indicates a tilted phase with no positional order within the layers, " $a$ " that the tilt order is anticlinic (or alternating), and "**" means that the phase is chiral. The anticlinic arrangement of chiral molecules gives the phase antipolar and antiferroelectric properties.

AFLCs are attractive for a variety of electro-optic applications. In ALFC displays the $\mathrm{SmC}_{a}^{*}$ material is confined between two glass plates, separated by a constant distance of a few micrometers. The smectic layers are arranged perpendicular to the glass plates (bookshelf geometry) in a unique direction, which produces a birefringent slab with its slow axis along the smectic layer normal. The AFLC cell is placed with the smectic layers parallel and perpendicular to crossed polarizers. An electric field $\mathbf{E}$ applied perpendicular to the plates switches the liquid crystal from the anticlinic antiferroelectric state at $\mathbf{E}=\mathbf{0}$ (dark) to one of the two symmetric synclinic ferroelectric states (bright) depending on the polarity of the field. AFLC displays have a number of attractive features such as intrinsic dc compensated drive, video speed capability, gray scale, and very wide viewing angle. However, these displays, despite a number of promising developed prototypes, have never reached the market and the main reason for this is the relatively low contrast achieved so far.

The contrast problem in AFLC displays is related to the limited extinction (light leakage) in the dark state. AFLC materials are notoriously difficult to align and form "horizontal chevrons" in which the layer normal (coinciding with the local slow axis of the optical indicatrix) varies across the surface of the sample. This results in static light leakage between crossed polarizers. Furthermore, in passive addressing, nonselected pixels do not experience $\mathbf{E}=\mathbf{0}$ but rather a voltage approximately equal to the holding voltage. This gives a dynamic light leakage due to the so-called pretransitional effect, which is a weak linear electro-optic effect active at applied fields below the threshold for switching to the ferroelectric state.

We have earlier shown that the static dark state problem can be solved by using a surface-stabilized orthoconic AFLC material (director tilt $\theta= \pm 45^{\circ}$ ) which gives a perfect dark state between crossed polarizers, independent of the quality of alignment [2,3]. Furthermore, it turns out that also the pretransitional effect, and thus the dynamic light leakage, is minimized or even completely absent under these conditions. In this paper, we show that both of these favorable results are directly connected to the correct orientation of the tilt plane (see below) of the AFLC structure with respect to the cell surfaces and the direction of the electric field. It is therefore of great importance for the development of future highperformance AFLC devices to know the internal structure of AFLC cells and, in particular, to be able to control the tilt plane orientation. We discuss how this orientation, under certain circumstances, can be determined by optical microscopy and dielectric measurements. In the latter case, the internal structure of the AFLC cell turns out to have a profound influence on the dielectric spectrum recorded. As a spin-off result, we can use dielectric spectroscopy to determine the degree of surface stabilization as a function of the history of the sample, which might be useful within the area of electronic addressing of AFLCs. As will be discussed later, it is of crucial importance for high contrast performance that the sample relaxes back completely to the horizontally surface stabilized (HSS) state after the field is removed. Finally, we discuss the field effects on the recently discovered antiferroelectric twist grain boundary phase $\left(\mathrm{TGBC}_{a}\right)$ [4]. It turns out that the electro-optic behavior of this complicated structure is well described based on the orientational response of the local tilt plane under electric fields.

\section{EXPERIMENT}

The AFLC cells are made of ITO-coated glass on which suitable electrode patterns were created by means of photo- 
lithography and wet etching. For planar anchoring we covered the inner surfaces with a polyimide (Pyralin) by means of spin coating. After hardening at elevated temperature, the surfaces were buffed with a velvet cloth, the cells were assembled, and the glass plates were fixed together with uvcuring glue (NOA 68). The cell thickness was assured by spacer balls mixed into the glue. The cells were filled with the liquid crystal materials by means of capillary forces in the isotropic state. Optic and electro-optic measurements were performed in a Nikon Optiphot polarizing microscope equipped with a photodetector, alternatively a camera. The applied fields were supplied by means of a Leader function generator and an FLC Electronics high-frequency amplifier, and monitored, together with the transmitted light intensities, on a Tektronix TDS 340 oscilloscope. The sample temperature was controlled using a Mettler FP 5 temperature control unit and a FP52 hot stage. Dielectric measurements were performed on the same type of sample using an HP 4192A dielectric bridge.

\section{THE TILT PLANE AND ELECTRIC FIELDS}

Let us first consider the achiral $\mathrm{SmC}_{a}$ phase, most easily observable in a racemic mixture. The tilt plane is defined as the plane spanned by the director and the smectic layer normal, cf. Fig. 1(a). Such an anticlinic structure is generally biaxial, with the two optic axes lying in a plane perpendicular to the tilt plane, Fig. 1(b). Its mesoscopically averaged dielectric tensor takes the form

$$
\varepsilon=\left(\begin{array}{ccc}
\varepsilon_{x} & 0 & 0 \\
0 & \varepsilon_{y} & 0 \\
0 & 0 & \varepsilon_{z}
\end{array}\right),
$$

where the $x$ direction is parallel to the tilt plane and perpendicular to the smectic layer normal $z$, and the $y$ direction is perpendicular to the tilt plane. The mesoscopic and macroscopic dielectric permittivities (in the achiral case these are the same) are given by

$$
\begin{gathered}
\varepsilon_{x}=\varepsilon_{1} \cos ^{2} \theta+\varepsilon_{3} \sin ^{2} \theta, \\
\varepsilon_{y}=\varepsilon_{2}, \\
\varepsilon_{z}=\varepsilon_{1} \sin ^{2} \theta+\varepsilon_{3} \cos ^{2} \theta,
\end{gathered}
$$

where $\varepsilon_{1}, \varepsilon_{2}$, and $\varepsilon_{3}$ are the principal permittivities in the local director frame [2]. For moderate tilt $\left(\theta<30^{\circ}\right)$ it turns out that $\varepsilon_{y}<\varepsilon_{x}<\varepsilon_{z}$ at optical frequencies, in conformity with Fig. 1(b).

In the chiral case $\left(\mathrm{SmC}_{a}^{*}\right)$, there is a helical structure superposed on the local anticlinic structure as an effect of the molecular chirality. As a result, the structure is mesoscopically biaxial but macroscopically uniaxial. In the helical structure, the directors of adjacent layers and the smectic layer normal no longer lie in a plane. The director rotates around the layer normal, on the surface of a cone, the angle $\delta \phi=180^{\circ}-\alpha$ between adjacent smectic layers, as schematically illustrated in Fig. 1(c). The angle $\alpha$ is of the order of 1

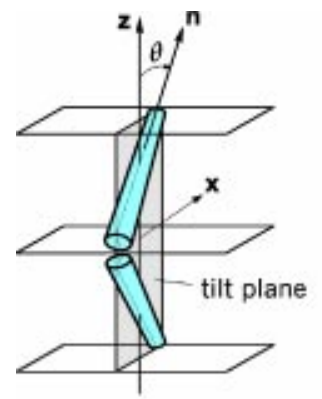

a)

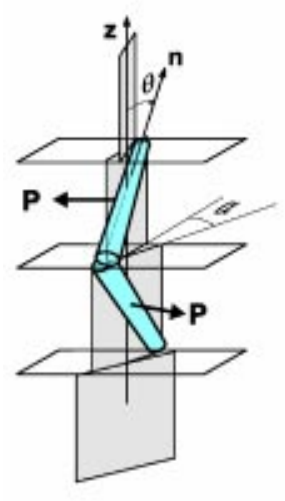

c)

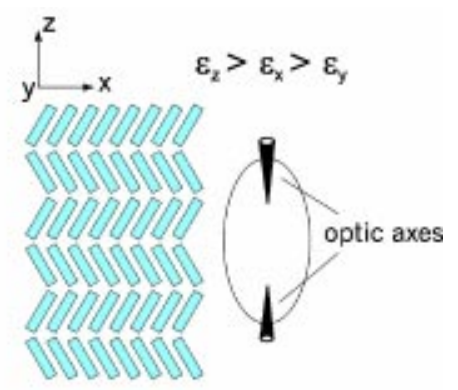

b)

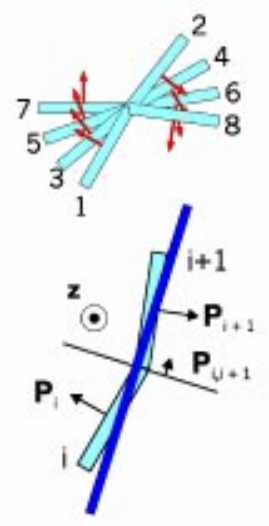

d)
FIG. 1. (a) Definition of the tilt plane in the anticlinic structure of the achiral $\mathrm{SmC}_{a}$ phase. (b) Illustration of the optical indicatrix of the biaxial structure of an anticlinic material. The two optic axes are in a plane perpendicular to the tilt plane. (c) The local structure of the helicoidal chiral $\mathrm{SmC}_{a}^{*}$ phase. (d) The definition of the local "tilt plane" in $\mathrm{SmC}_{a}^{*}$ used in this paper.

degree. The helical structure furthermore has the effect that the electric dipoles of neighboring layers do not cancel but change direction in the same helical fashion. Macroscopically there is no net polarization as the dipoles sum up to zero for every half helical period. (In the corresponding synclinic case, we have to sum over the full helical period to achieve complete cancellation.)

When an electric field is applied perpendicular to the layer normal of such a double helix structure, the directors in every other layer tend to turn clockwise, in alternate layers anticlockwise, as the local $\mathbf{P}_{s}$ wants to orient along the field. This tends to increase the angle $\alpha$ between successive oddeven layer pairs and to decrease it between the even-odd pairs, to equal degree counteracted by the helical elasticity and, in particular, by nearest-neighbor interaction tending to preserve the anticlinic tilt in a plane. As a result, a local $\mathbf{P}_{s}$ cannot move into the field without its neighbors moving out of it. As the field distorts the helix, a macroscopic polarization appears. This is a dielectric effect not related to the dielectric anisotropy as in the cholesteric case but to the coupling between the electric field and the local spontaneous polarization. However, due to the anticlinic constraint, the final (unwound) state is such that no dipole of a single layer points in the direction of the field, only the residual dipole from a layer pair. 


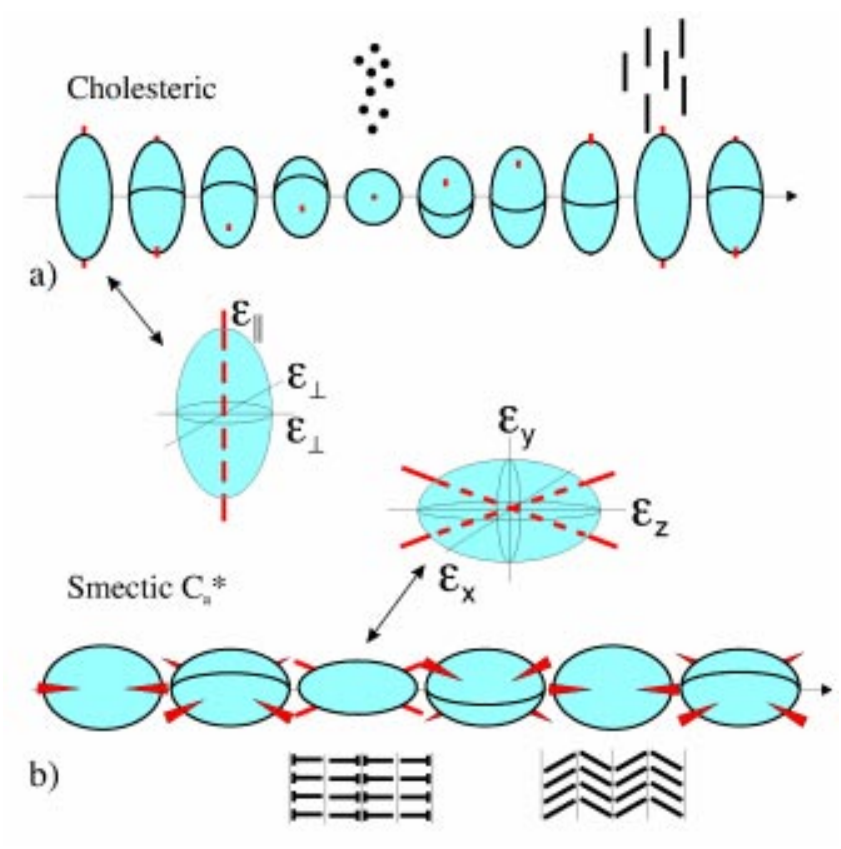

FIG. 2. The spiraling index ellipsoids of (a) the cholesteric phase and (b) the antiferroelectric $\operatorname{SmC} C_{a}^{*}$ phase. In (a) the local structure is uniaxial, in (b) it is biaxial, cf. Fig. 1(b).

If we look at the initial helicoidal structure under the applied field and pay attention to the set of odd-numbered layers, we find that for every period corresponding to half the pitch, some layers are favorably oriented with respect to the applied field. Their even-numbered neighbors are in the same degree particularly disfavored energetically. Furthermore, in the next half period the situation is reversed. It is therefore convenient to regard pairs of nearest neighbors of layers and study how their resultant $\mathbf{P}$ vector behaves under the field. For simplicity, we can relate this polarization to each layer interface (between layer $i$ and layer $i+1$ ) and we may denote it

$$
\mathbf{P}_{i, i+1}=\mathbf{P}_{i}+\mathbf{P}_{i+1} .
$$

Note that $\mathbf{P}_{i, i+1}$ is essentially pointing in opposite directions at adjacent layer interfaces, i.e., for odd and even values of $i$. In order to describe the helical anticlinic structure in a convenient way, we will for simplicity still use the term "tilt plane," now referring to the plane spanned by the layer normal and the direction of the net polarization $\mathbf{P}_{i, i+1}$ of each layer pair, cf. Fig. 1(d). Thus we may describe the helical $\mathrm{Sm} C_{a}^{*}$ phase as an anticlinic structure where the tilt plane is slowly rotating about the smectic layer normal and, as we will see below, the helix unwinding is conveniently described by the action on this tilt plane. The structure is locally biaxial and we can, to a good approximation, also describe the $\mathrm{SmC}_{a}^{*}$ phase by a spiraling index ellipsoid with the $x$ and $y$ axes always perpendicular to the helix axis as illustrated in Fig. 2. In helix-free $\mathrm{SmC}_{a}^{*}$ samples, where the helix is unwound by cell surfaces, or in pitch-compensated mixtures $(p=\infty)$ the tilt plane is identical to that of a nonchiral $\mathrm{SmC}_{a}$ phase with the dielectric tensor given by Eq. (1).

Gorecka et al. studied the antiferroelectric liquid crystal MHPOBC in thick freely suspended films by means of conoscopy under the action of electric fields applied in the plane of the film, thus parallel to the smectic layers and perpendicular to the helix axis [5]. At zero field they observed a centered extinction cross typical for a uniaxial crystal with its optic axis perpendicular to the film. On increasing the electric field, the evolution of the conoscopic picture revealed that the film became biaxial as the helix started to unwind with the two optic axes in a plane perpendicular to the field direction. This is a general feature of AFLCs and shows that the tilt plane of the unwound (helix-free) structure of antiferroelectric liquid crystals aligns along the field direction. Note that the maximum applied field in the film experiment by Gorecka et al. $(1200 \mathrm{~V} / \mathrm{mm})$ is much lower than the threshold field $\mathbf{E}_{\mathrm{th}}$ required for switching the MHPOBC sample into the ferroelectric state $\left(\mathbf{E}_{\mathrm{th}} \approx 12000 \mathrm{~V} / \mathrm{mm}\right)$. Furthermore, the conoscopic picture indicated that the optic indicatrix was slightly tilted in a plane perpendicular to the field direction.

Recently, it was shown how the helix profile of an AFLC distorts when subjected to an electric field perpendicular to the helix axis [6,7]. A theoretical analysis of the conoscopic picture developing during the unwinding was made by Roy and Madhusudana [8,9]. The unwinding of the AFLC helix is similar to, but shows a characteristic difference from, the field-induced unwinding of a cholesteric helix. In the latter case, the unwinding is due to the dielectric coupling to the field $\left(\varepsilon_{\|}>\varepsilon_{\perp}\right)$, which makes the director (the axis of highest permittivity) align along the field [10]. At first sight, one could imagine a similar dielectric effect being responsible for the helix unwinding in the AFLC case, provided that the axis of highest permittivity along the smectic layer is in the tilt plane, i.e., if $\varepsilon_{x}=\varepsilon_{1} \cos ^{2} \theta+\varepsilon_{3} \sin ^{2} \theta>\varepsilon_{y}=\varepsilon_{2}$. [Here $\varepsilon_{i}(i$ $=1,2,3$ ) are the principal low-frequency dielectric permittivities in the local molecular frame.] But this dielectric effect is totally obscured by the much stronger coupling of the electric field to the permanent electric dipoles of the smectic layers, illustrated in Fig. 3. Provided that the anticlinic order is sufficiently stable, such that there is no switching to the synclinic ferroelectric state at this level of applied field, the helical structure totally vanishes at a certain field strength, giving a helix-free antiferroelectric structure with "the tilt plane along the field." Note, however, that the plane containing the directors of adjacent layers is somewhat tilted with respect to the smectic layer normal in the field-induced unwound state, as illustrated in Fig. 3. This explains that the optical indicatrix in the unwound state is slightly tilted out perpendicular to the field direction. On further increasing the applied field, the switching to the synclinic state finally occurs at a certain field threshold.

\section{OPTICAL AND DIELECTRIC STUDIES OF THE TILT PLANE ORIENTATION}

We want to relate the internal structure, or the tilt plane orientation of AFLC cells to the electro-optic performance 

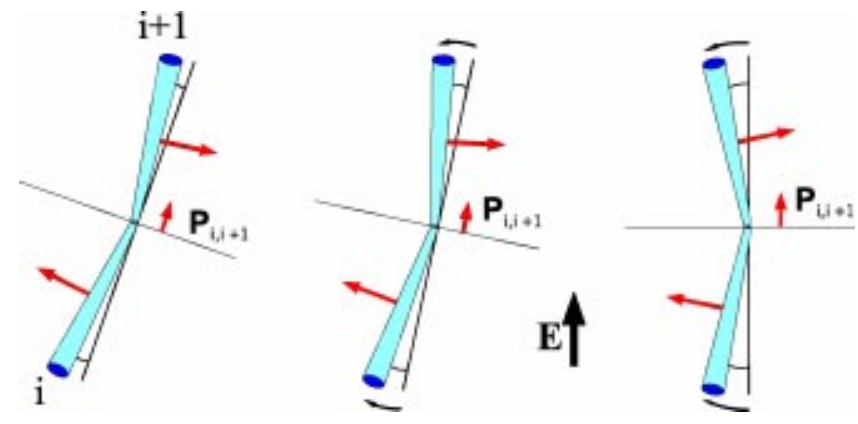

FIG. 3. Field-induced reorientation of the tilt plane due to the torque $\mathbf{P} \times \mathbf{E}$, where $\mathbf{P}=\mathbf{P}_{i, i+1}$ and $\mathbf{E}$ is the applied electric field perpendicular to the smectic layer normal. Note that $\mathbf{E}$ further increases the antiphase distortion of the anticlinic structure in the process (evolution from left to right). In the figure, the smectic layers are parallel to the plane of the paper. In the final state (right) of the helix unwinding, all unit cells have aligned with the tilt plane in the direction of the field. Note that the individual $\mathbf{P}_{s}$ pairs are almost perpendicular to $\mathbf{E}$. Only their resultant is along the field.

and the recorded dielectric relaxation spectra. It is of particular interest to be able to conclusively distinguish the HSS condition from other configurations. Therefore, we need to compare the dielectric spectra for samples which are unambiguously horizontal surface stabilized with samples which are not. The first step is to optically determine when the AFLC is in the HSS state.

\section{A. Optical investigations}

The principal refractive indices of a helix-free AFLC material is given by [2]

$$
\begin{gathered}
n_{x}=\sqrt{n_{1}^{2} \cos ^{2} \theta+n_{3}^{2} \sin ^{2} \theta}, \\
n_{y}=n_{2}, \\
n_{z}=\sqrt{n_{1}^{2} \sin ^{2} \theta+n_{3}^{2} \cos ^{2} \theta} .
\end{gathered}
$$

Provided that the cell thickness $d$, the tilt angle $\theta$, and the principal refractive indices $n_{1}, n_{2}$, and $n_{3}$ of the synclinic state are well known, one can make a coarse estimation of the internal structure of a bookshelf AFLC cell from the measured optical relative retardation of the cell. In an HSS cell, the tilt plane is everywhere parallel to the cell plane [Fig. 4(a)] and the retardation at normal incidence is $2 \pi d\left(n_{z}-n_{x}\right) / \lambda$, where $\lambda$ is the wavelength of light in vacuum. If instead the tilt plane is essentially vertical, cf. Fig. 4(b), the retardation is $2 \pi d\left(n_{z}-n_{y}\right) / \lambda$. If neither of these two situations apply, e.g., in the helical state, or if the tilt plane is inclined with respect to the glass plates, the retardation takes some intermediate value. This method is, however, not very accurate. We have therefore chosen to use orthoconic antiferroelectric liquid crystals, which have a molecular tilt of $45^{\circ}$. Such materials are locally uniaxial with the optic axis perpendicular to the tilt plane. Therefore, an HSS orthoconic AFLC material looks perfectly black between crossed polarizers, independent of the direction of the smectic layer normal. This unique property directly reveals

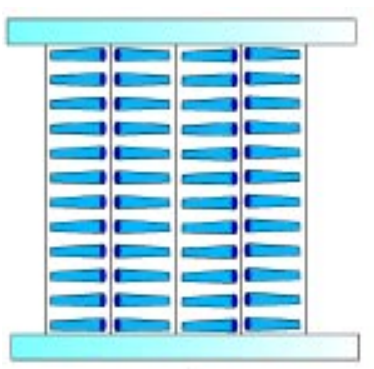

a)

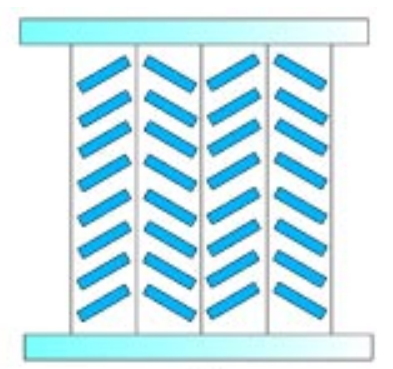

b)
FIG. 4. Schematic illustration of (a) horizontally surfacestabilized (HSS) structure and (b) vertical tilt plane structure.

when the orthoconic AFLC is in the HSS state. Furthermore, we do not need to know the values of the principal refractive indices nor the cell thickness. By using such cells, we have been able to make systematic studies of the electro-optic and dielectric response of AFLC cells in order to relate it to the tilt-plane orientation.

An illustration of the unique properties of orthoconic AFLCs is given in Figs. 5(a) and 5(b) where the electro-optic switching of two surface-stabilized cells, with the material W107 [11], is shown. The two microphotographs to the left show the zero-field anticlinic state, which in both cells is black, despite the large variations of the smectic layer orientation in the cell in Fig. 5(b), compared to the well-aligned cell in Fig. 5(a). In Fig. 6, the coexistence of ferroelectric and antiferroelectric domains is shown, which easily occurs in thin cells [12]. The crossed polarizers are parallel to the edges of the photograph. In Fig. 6(a), the antiferroelectric domains are black and the ferroelectric domains $\left(\mathbf{P}_{s}\right.$ UP and $\mathbf{P}_{s}$ DOWN domains) appear white. When rotating the cell [Fig. 6(b)], a major part of the black antiferroelectric do-

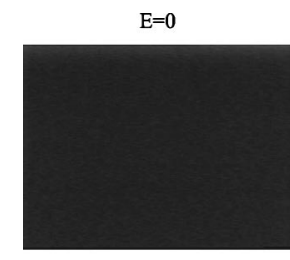

$\mathrm{E}=0$
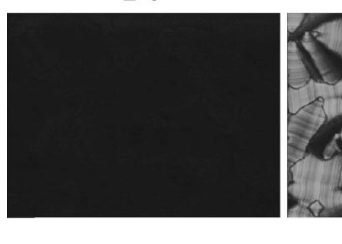

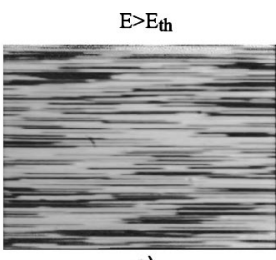

a)

$\mathrm{E}>\mathrm{E}_{\text {th }}$

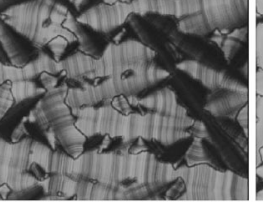

b)
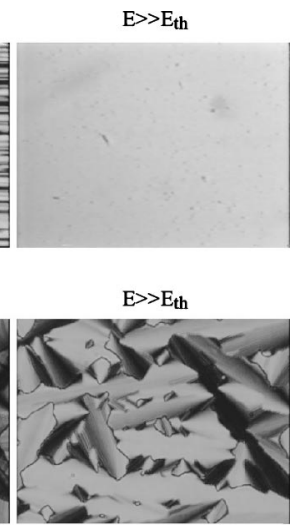

FIG. 5. Electro-optic switching in horizontally surface-stabilized orthoconic $\left(\theta=45^{\circ}\right)$ AFLC cells studied between crossed polarizers which are parallel to the edges of the pictures. The zero field state is completely black both in the well-aligned (a) and the random aligned (b) bookshelf structure. This is due to the fact that the optic axis is everywhere perpendicular to the glass plates, irrespective of the orientation of the smectic layer normal in the plane of the cell. The random alignment of the cell in (b) is evident in the partly and fully field-induced synclinic state. 


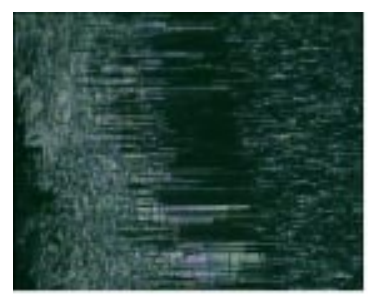

a)

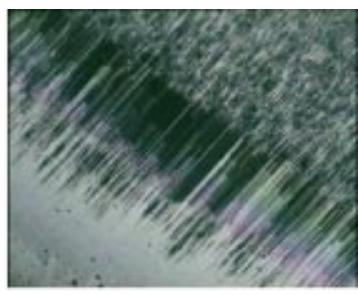

b)
FIG. 6. Microphotographs of an $\sim 0.5$ - $\mu$ m-thick orthoconic AFLC bookshelf cell between crossed polarizers. On rotating the cell, we can immediately recognize which domains are in the horizontally surface-stabilized state, as they appear black for any position of the cell between crossed polarizers. In (a) the cell is in the standard AFLC display geometry with the smectic layers horizontal in the picture along the polarizer. In (b) the cell is rotated $45^{\circ}$ from this position.

mains stays black, which reveals that this part is HSS. But there are also antiferroelectric areas that now look bright, which indicates that they are not HSS. Due to the optical properties of orthoconic AFLCs, we can immediately see which parts of the cell are HSS and which are not.

The texture of Fig. 6 was obtained after cooling down from the synclinic $\mathrm{SmC}^{*}$ phase. A similar texture, with coexisting domains of ferroelectric and antiferroelectric domains of different structure, can appear in thin cells also after switching to the ferroelectric states. (Generally, the sample has to be electrically addressed using dedicated waveforms in order to fully relax back to the antiferroelectric state when the field is switched off. For triangular voltages, this type of texture may give complicated transmission-voltage curves as the different domains switch at different applied voltages, which can be misinterpreted as ferrielectric behavior [13].

\section{B. Dielectric investigations}

Let us now look at the dielectrically active modes in AFLCs. Three of these are schematically illustrated in Fig. 7. (a) Molecular reorientation around the short axis, in the following simply referred to as "short axis fluctuations." This mode $\left(f_{c} \sim \mathrm{kHz}-\mathrm{MHz}\right)$ appears in the dielectric spectrum when the measuring field $\mathbf{E}_{m}$ has components along the director. Thus, it is absent if $\mathbf{E}_{m}$ is strictly perpendicular to the tilt plane. (b) Antiphase fluctuations $\left(f_{c} \sim 100 \mathrm{kHz}\right)$ of the molecules of adjacent smectic layers giving polarization fluctuations in the tilt plane, perpendicular to the smectic layer normal. This mode appears in the spectrum when $\mathbf{E}_{m}$ has a component in that direction but it does not appear when $\mathbf{E}_{m}$ is applied strictly perpendicular to the tilt plane or strictly parallel to the smectic layer normal, cf. Fig. 9. (c) Tilt plane fluctuations $\left(f_{c} \sim \leqslant \mathrm{kHz}\right)$. This mode appears when the measuring field is applied neither parallel nor perpendicular to the tilt plane. In a helical sample, this can be regarded as a helix distortion mode. The tilt plane fluctuation mode may also appear in surface-stabilized samples in connection with the AFLC Frederiks transition, as will be discussed below.

There are, in principle, two other dielectrically active modes in AFLCs that are not discussed here: (i) the antitilt mode, which is a fluctuation increasing and decreasing the tilt by the same amount in adjacent smectic layers with an "electrocliniclike" coupling between tilt and polarization in each layer, and (ii) the noncollective fluctuations around the molecular long axes. Mode (i) appears essentially only in the vicinity of a $\operatorname{Sm} C_{a}^{*}$ to $\operatorname{Sm} A^{*}$ transition and is very weak far from this transition. Furthermore, if present here it is likely to be obscured in our measurements by the dielectric relaxation of the measurement cell itself. However, as we cannot see any optical response in HSS cells below the F to AF transition, we argue that the amplitude of this anticlinic "electrocliniclike mode" is very small and can be neglected far from the $\operatorname{Sm} A$ * phase. Mode (ii) has a critical frequency in the $\mathrm{GHz}$ region and does not appear in the dielectric measurements performed here $(5 \mathrm{~Hz}-13 \mathrm{MHz})$.

The discussion in relation to Fig. 7 makes it obvious that the appearance of a particular mode in the $\mathrm{SmC}_{a}^{*}$ dielectric
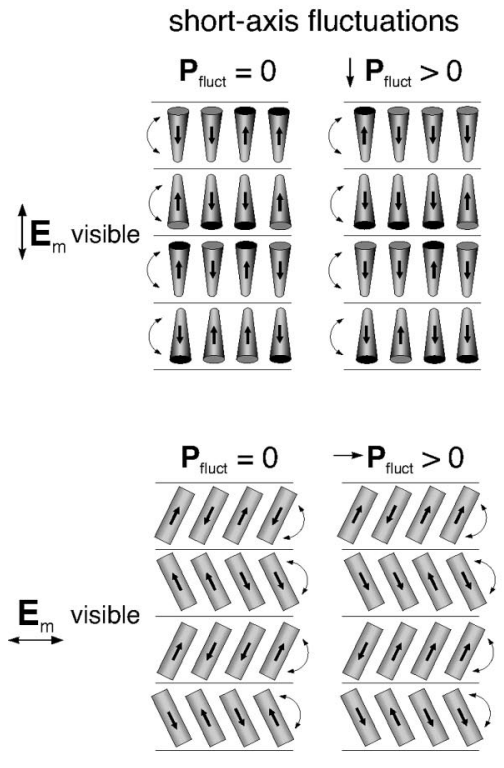

a)
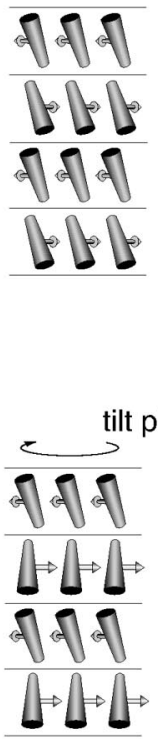

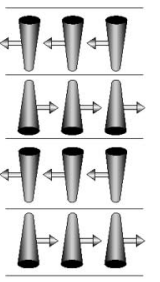

b)

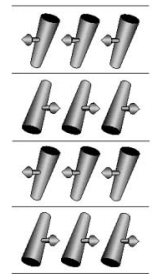

$\bullet \mathbf{E}_{\mathrm{m}}$ visible

plane fluctuations
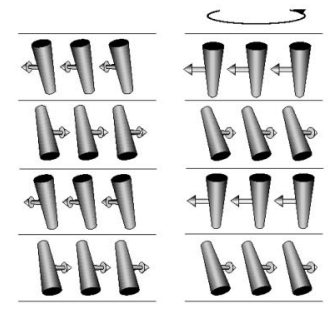

FIG. 7. Schematic illustration of the active dielectric modes, showing for which directions of the measuring field they contribute to the dielectric permittivity, i.e., they are visible in the dielectric spectrum. The tilt plane fluctuations are visible only when there is an induced polarization in the tilt plane, e.g., under a dc bias or when the measuring field is applied at an oblique angle to the tilt plane. In the latter case, the polarization in the tilt plane is induced by the measuring field. Note that when the measuring field is applied normal to the tilt plane, none of the modes contribute to the measured permittivity. 


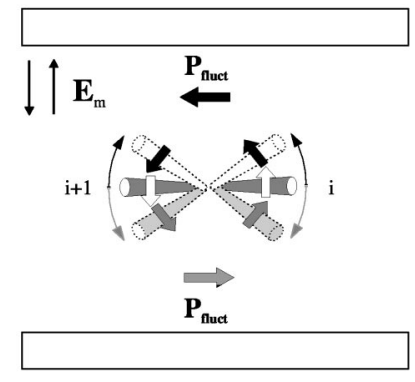

a)

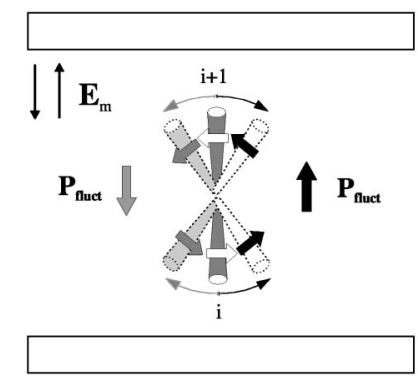

b)
FIG. 8. The antiphase fluctuation mode gives a fluctuating polarization $\mathbf{P}_{\text {fluct }}$ in the tilt plane. Thus in dielectric spectroscopy, when the measuring field is applied perpendicular to the glass plates, there is no contribution from the antiphase fluctuations in the horizontally surface stabilized state (left, $\mathbf{P}_{\text {fluct }} \perp \mathbf{E}_{m}$ ) whereas there is a maximum contribution in the vertical tilt plane state (right, $\left.\mathbf{P}_{\text {fluct }} \| \mathbf{E}_{m}\right)$. In the figure the smectic layers are parallel to the plane of the paper and the molecules in layers $i$ and $i+1$ are depicted.

spectrum is strongly dependent on the orientation of the tilt plane. Figure 8 shows a schematic picture of a conventional sandwich cell used for dielectric spectroscopy where the measurement field is applied between the electroded glass plates. We first assume that we have a bookshelf aligned AFLC material with the smectic layers in the plane of the paper, and consider the case of horizontal surface stabilization, cf. Figs. 4(a) and 8(a). The fluctuating polarization $\mathbf{P}_{\text {fluct }}$ due to antiphase fluctuations is now parallel to the glass plates and normal to the measurement field. Thus, there is no contribution to the dielectric permittivity measured across the cell and the antiphase mode does not appear in the spectrum. If we instead consider a vertical tilt plane situation
TABLE I. The AFLC dielectric modes.

\begin{tabular}{lcccc}
\hline \hline Mode & Homeotropic & $\begin{array}{c}\text { Bookshelf } \\
\text { horizontal } \\
\text { tilt plane }\end{array}$ & $\begin{array}{c}\text { Bookshelf } \\
\text { vertical } \\
\text { tilt plane }\end{array}$ & $\begin{array}{c}\text { Bookshelf } \\
\text { helical } \\
\text { structure }\end{array}$ \\
\hline Antiphase & invisible & invisible & visible & visible \\
Short axis & visible & invisible & visible & visible \\
Tilt plane & invisible & invisible & invisible & visible \\
Long axis & visible & visible & visible & visible \\
Antitilt & invisible & visible & invisible & visible \\
\hline \hline
\end{tabular}

[Figs. 4(b) and 8(b)] the fluctuating electric polarization is along the electric field and gives a strong contribution to the spectrum. We can thus conclude that the strongest contribution to the dielectric permittivity from the antiphase fluctuations would emerge from a uniform vertical tilt plane structure [7].

The AFLC dielectric modes, along with the geometries in which they appear, are summarized in Table I. Note that in the bookshelf geometry, tilt plane fluctuations, short-axis fluctuations, and antiphase fluctuations (these three modes are likely the modes 0,1 , and 2 discussed in [14]) can all appear together. Moreover, the tilt plane fluctuations are closely linked to the antiphase fluctuations: the visibility of the former requires a polarization in the tilt plane, which is induced by the latter. As can be seen in Table I, none of the three mentioned modes appears in the case of a horizontal tilt plane. In the case of a vertical tilt plane, the short-axis and antiphase fluctuations exhibit their strongest response, while the tilt plane fluctuation does not contribute. The tilt plane mode is generally visible in the presence of a dc bias field of intermediate strength [14]. In conclusion, the dielec-

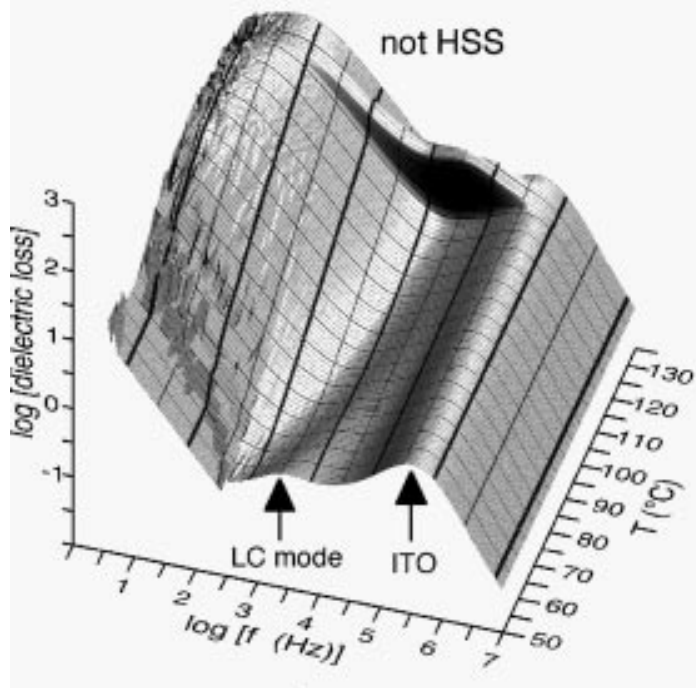

a)

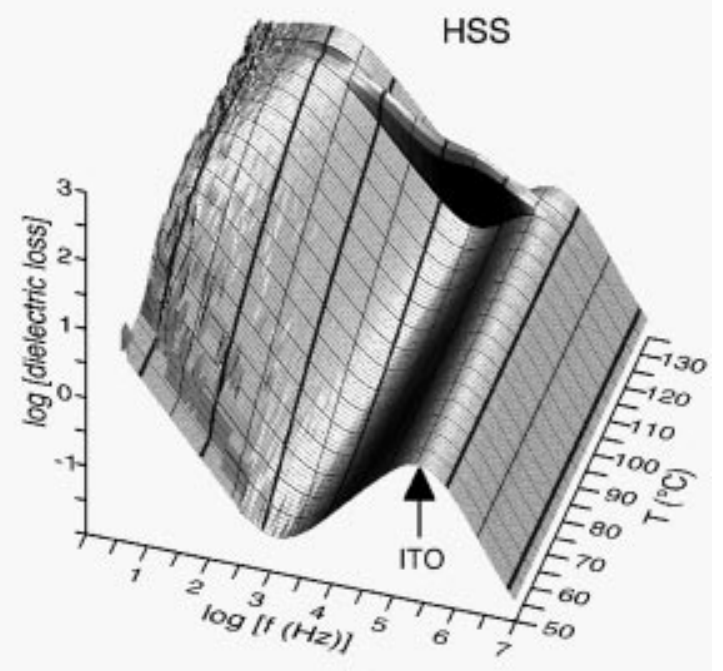

b)

FIG. 9. Dielectric loss spectra of the orthoconic AFLC material W107 on heating. (In order to avoid perspective inversion when looking at the picture, note that the illumination comes from the right and thus the ITO peak marked with an arrow goes up/out of the paper.) In (a) the material is not horizontally surface stabilized and there is a mode appearing in the spectrum. In (b) the same sample is horizontally surface stabilized and there is no contribution from this mode anymore. The strong mode above $125^{\circ} \mathrm{C}$ is the collective phase angle fluctuation mode in the synclinic SmC* phase. In the labels $\log$ corresponds to $\log _{10}$. 
tric spectrum gives much information about the quality of the surface-stabilized state.

Figure 9 shows two dielectric absorption spectra, both taken on heating, of an approximately $0.5-\mu \mathrm{m}$-thick cell with the orthoconic AFLC material W107 having the phase sequence

$$
\text { Isotropic } 135^{\circ} \mathrm{C}-\mathrm{Sm} A^{*}-132{ }^{\circ} \mathrm{C}-\mathrm{Sm} C^{*}-125^{\circ} C \text {-Sm } C_{a}^{*} \text {. }
$$

The first measurement [Fig. 9(a)] was taken after cooling from the isotropic phase to the $\mathrm{SmC}_{a}^{*}$ phase with no electric addressing of the cell before the measurement. As the $\mathrm{SmC}_{a}^{*}$ texture was not black for all positions of the cell between crossed polarizers, but rather inhomogeneous in brightness, we could conclude that it was not in the HSS state. This is supported by the recorded spectrum in which a lowfrequency $\left(f_{c} \sim 3.5 \mathrm{kHz}\right)$ mode is clearly visible in the antiferroelectric phase. (Modes at frequencies higher than $\sim 10^{5} \mathrm{~Hz}$ cannot be studied due to the low cutoff frequency of a cell this thin.) The sample was then cooled down again and electrically addressed until a perfect black state was achieved for all rotations between crossed polarizers. The same measurement was repeated and now there was, as expected, no trace of any dielectric mode. This is thus an experimental verification that the horizontal surface-stabilized condition of AFLCs can be probed by means of dielectric spectroscopy. This holds also for conventional $\mathrm{SmC}_{a}^{*}$ materials with tilt angles of about $30^{\circ}$ where the horizontal condition cannot easily be detected optically. In order to identify the observed mode, further analysis has to be performed. It is likely that the high-frequency antiphase mode has a critical frequency close to or above the cutoff frequency of the cell. Electro-optic investigations should reveal if the observed mode is short-axis fluctuations (not electro-optically active) or tilt-plane fluctuation (electro-optically active).

\section{Frederiks transition}

As theoretically predicted by Qian and Taylor [15] and recently experimentally verified by Wen $e t a l$. and Zhang et al. [16], a horizontally surface-stabilized AFLC material may undergo a Frederiks transition to a vertical tilt plane state before switching to the synclinic ferroelectric state. When the electric field increases from zero, the tilt plane is at first parallel to the glass plates and there is no torque on the local polarization of the smectic layers. For low voltages, fluctuations in the tilt plane orientation are counteracted by the elastic torques imposed by the planar anchoring at the surfaces. However, above a certain threshold field, proportional to $1 / d$, any fluctuation of the tilt plane orientation will result in a local torque strong enough to break the HSS structure. The tilt plane starts to reorient away from the cell plane, similar to the way in which the director tilts out of the layer plane in the Frederiks transition of a planar aligned nematic, until the sum of the electric and elastic energies is minimized. As the field increases, a larger and larger part of the bulk will have the tilt plane along the electric field. The active torque on the tilt plane in this Frederiks transition is the same as in the field-induced unwinding of the AFLC helix discussed earlier, i.e., the coupling of the noncanceling
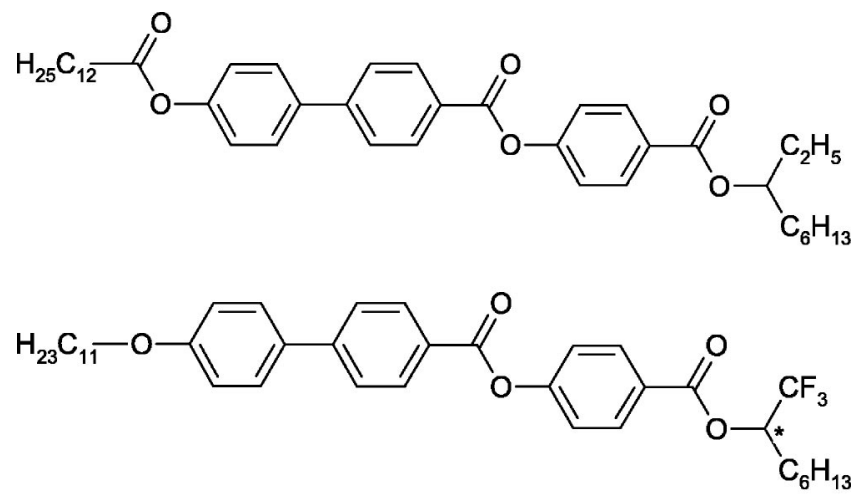

FIG. 10. Chemical structures of (a) (S)-TFMHPnBC-11 and (b) (S)-EHPnCBC-12.

polarization coming from the field-induced antiphase distortion of the anticlinic structure. Thus, the $x$ axis of the biaxial dielectric tensor of the surface-stabilized AFLC reorients in the electric field like the director of a nematic liquid crystal in the corresponding Frederiks transition. Moreover, as in all Frederiks transitions in this type of geometry, the threshold field is inversely proportional to the cell thickness, i.e., it is rather a voltage threshold than a field threshold.

We made a $2-\mu \mathrm{m}$ thick cell with planar anchoring filled with a 70:30 mixture of (S)-TFMHPnBC-11 and (S)EHPnCBC-12 (see Fig. 10). This AFLC mixture is nearly pitch-compensated (but not polarization-compensated) and gets essentially horizontally surface stabilized in $2-\mu \mathrm{m}$ cells.

We measured both the optical response due to an ac electric field and the dielectric response under a dc bias electric field, cf. Fig. 11. Part (a) in this figure shows the average transmitted light intensity as a function of the amplitude of the ac field when the cell is placed between crossed polarizers in the standard AFLC display geometry, i.e., with the crossed polarizers along and perpendicular to the average smectic layer normal direction. On increasing the electric field from zero, nothing happens until the Frederiks transition occurs at about $4 \mathrm{~V}$, where there is an increase of the transmitted signal. The increase in intensity is due to the effective tilt of the slow axis in the plane of the cell coming from the field-induced antiphase deflection of the molecules in adjacent layers. There is also a change in transmission due to the increase of the retardation when the tilt plane is rotated away from the plane of the cell. The magnitude of the former effect depends on the amount of rotation of the tilt plane and on the amplitude of the field, whereas the magnitude of the latter effect depends on the quality of the alignment of the smectic layer normal in the plane of the cell, as will be discussed later.

Note that there is no electro-optic effect below the Frederiks transition. At about $10 \mathrm{~V}$, equal to a field strength of about $5.0 \mathrm{~V} / \mu \mathrm{m}$, the transition from the antiferroelectric (AF) to the ferroelectric (F) state occurs. The combination of electro-optic effects below this threshold is generally referred to as the pretransitional effect. We will return to this matter in Sec. IV E. In Fig. 11(b), the dielectric absorption in the same cell as a function of frequency of the measuring field and amplitude of the bias field is shown. At zero bias, 


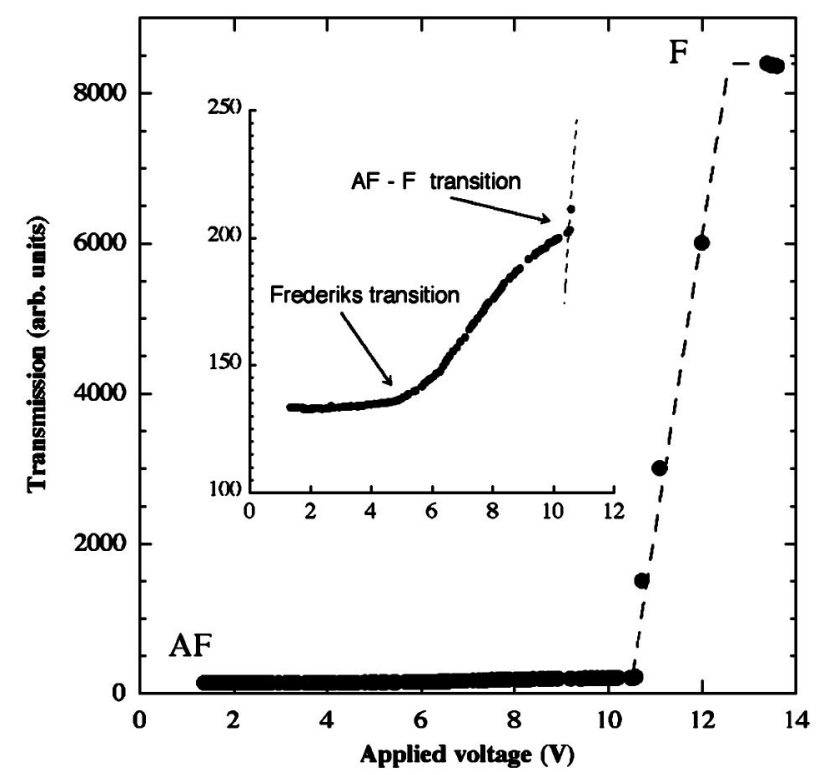

a)

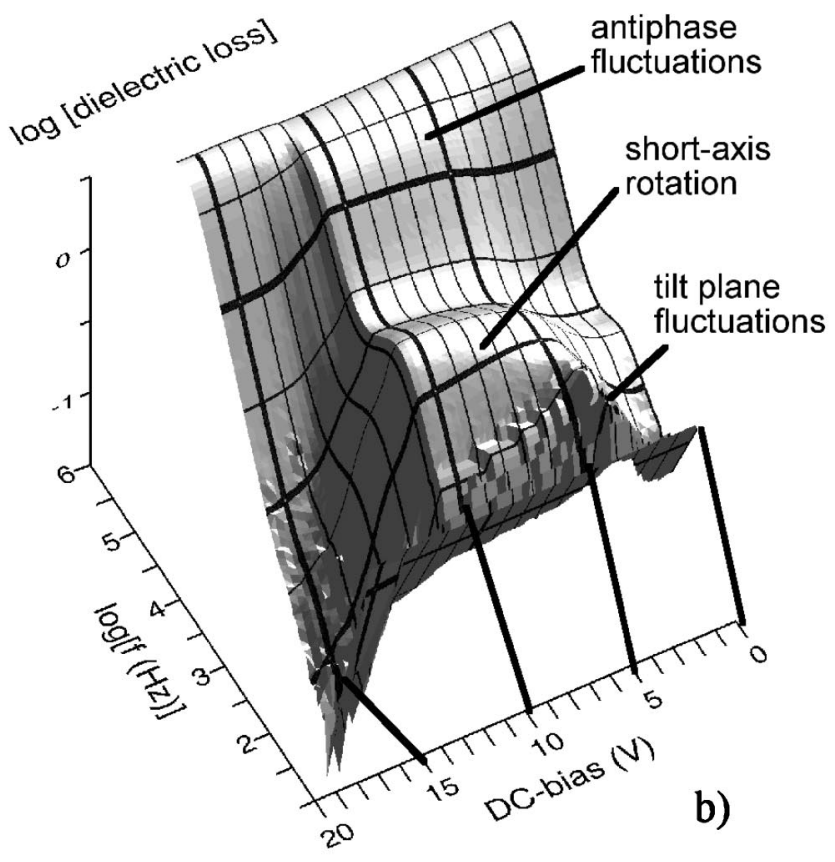

FIG. 11. The Frederiks transition in a horizontally surfacestabilized pitch-compensated AFLC material in a 2- $\mu \mathrm{m}$-thick cell studied by (a) electro-optic measurements and (b) dielectric spectroscopy. (a) shows the average transmitted intensity as a function of the amplitude of an $100 \mathrm{~Hz}$ ac electric field in standard AFLC display geometry. (b) shows the dielectric loss spectrum taken under the application of a bias voltage. The appearance of the three dielectric modes in (b) is directly related to the appearance of the pretransitional effect in (a) at $4.5 \mathrm{~V}$ when the Frederiks transition occurs, i.e., when the tilt plane starts to make an angle with the cell plane. For bias voltages between 5 and $10 \mathrm{~V}$, the tilt plane is essentially vertical. Finally, at $10 \mathrm{~V}$ bias $(5.0 \mathrm{~V} / \mu \mathrm{m})$, the transition to the ferroelectric state occurs giving a large increase in the transmitted intensity at the same time as the AFLC dielectric modes disappear. In the labels, log corresponds to $\log _{10}$.

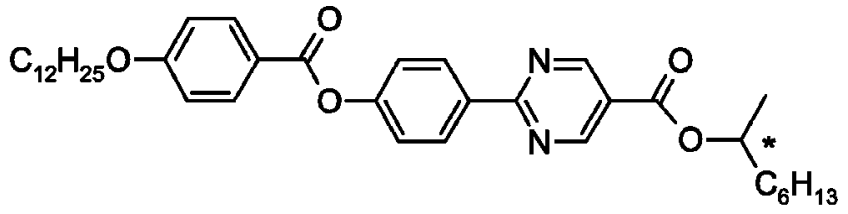

I $93.1^{\circ} \mathrm{C} \mathrm{SmQ} 92.9^{\circ} \mathrm{C} \mathrm{TGBC}$ a $91.6^{\circ} \mathrm{C} \mathrm{SmC}_{\mathrm{a}} 68.3^{\circ} \mathrm{C}$ m.p. $86.4^{\circ} \mathrm{C}$

FIG. 12. Molecular structure of the $\mathrm{TGBC}_{a}$ material.

only a very weak low-frequency mode $\left(f_{a} \sim 3 \mathrm{kHz}\right)$ appears. This indicates that the cell is essentially in the horizontal surface-stabilized condition but the alignment is not perfect over the entire electrode area. On increasing the bias voltage, there is a significant increase of this mode when the Frederiks transition occurs, i.e., when the tilt plane reorients from horizontal to vertical in the cell. The tilt plane reorientation itself appears as a third mode of very low frequency (the tilt plane fluctuation mode) visible for bias voltages in the range 3-5 V. Above $5 \mathrm{~V}$ dc bias, the tilt plane is essentially vertical, thus the mode disappears. When the tilt plane becomes vertical, also a high-frequency mode $(0.7 \mathrm{MHz})$ stands out from the ITO peak. This is the antiphase fluctuation mode. Note the sharp decrease of the dielectric loss as all AFLC modes disappear when the field-induced transition from the antiferroelectric to ferroelectric state occurs at about $5.0 \mathrm{~V} / \mu \mathrm{m}$. This experiment proves that what we see in the electro-optic measurements is in fact the Frederiks transition.

\section{Field effects in the antiferroelectric TGB phase}

Twist grain boundary (TGB) phases consist of smectic blocks forming a broken helical structure with the smectic layer normal of individual blocks spiraling about the helix axis. The blocks are separated by grain boundaries of parallel screw dislocations, which mediate the twist between the blocks. TGB phases appear when the helical twisting power is high enough to break the homogeneous layered structure of an ordinary smectic phase. The TGBA phase consists of smectic $A$ blocks, the TGBC phases consist of $\mathrm{Sm} C^{*}$ blocks, and so on. The TGBA phase was first predicted by Renn and Lubensky [17] and later discovered by Goodby et al. [18] while the TGBC phase was discovered by Nguyen et al. [19].

Recently, a new type of TGB phase with local anticlinic, antiferroelectric order within the smectic blocks was discovered in the compound depicted in Fig. 12 [4,20]. On the basis of optical and electro-optical investigations on the same compound, we developed a qualitative model for the microscopic organization of the $\mathrm{TGBC}_{a}$ phase [20]. In this model, the smectic layer normal of individual blocks is perpendicular to the TGB helix axis. Inside a block, the local tilt plane is parallel to the grain boundaries. The grain boundaries consist of a lattice of $+1 / 2$ and $-1 / 2$ dispirations, i.e., unit screw dislocations in the smectic layer structure combined with half unit disclinations in the $c$ director (the projection of the director onto the smectic layer). Whereas all screw dislocations have the same sign, the sign of the disclinations alternates in adjacent dispirations. A schematic picture of the structure proposed is given in Fig. 13. From the picture it is seen that within the grain boundaries the tilt plane between 


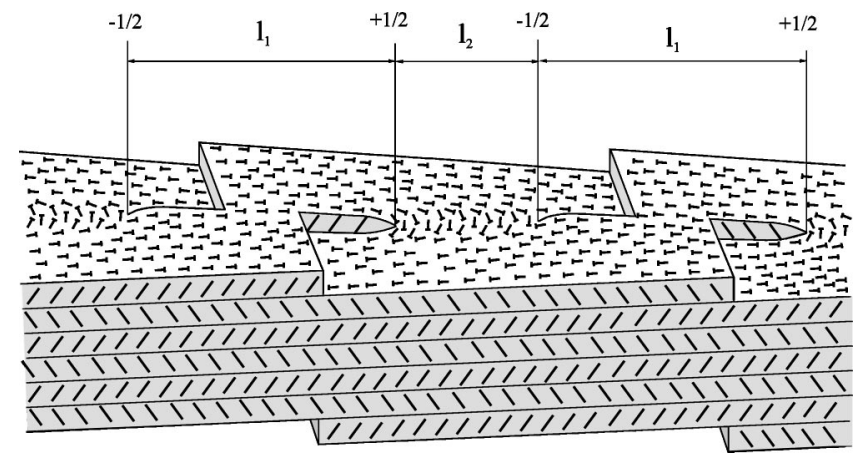

FIG. 13. The microscopic arrangement of the $\mathrm{TGBC}_{a}$ phase. The grain boundary consists of dispirations built up of unit screw dislocations combined with half unit disclinations.

adjacent dispirations is alternately parallel and perpendicular to the TGB helix.

The electro-optic effects of the $\mathrm{TGBC}_{a}$ phase can be summarized as follows. In planar aligned samples, the helix is perpendicular to the glass plates (Grandjean texture). An electric field applied normal to the cell walls first induces a slight increase of the helical pitch, detected as a change of the color of selective reflection. On increasing the field strength, there is a transition from Grandjean to fingerprint texture with the TGB helix parallel to the glass plates. For high enough fields, the TGB helix is unwound corresponding to a field-induced untwisted $\mathrm{SMC} C_{a}^{*}$ structure. All these features can be explained by considering the model depicted in Fig. 13 together with the basic knowledge that the local tilt plane wants to align along the applied electric field. In the Grandjean texture, the electric field is applied perpendicular to the tilt plane inside the smectic blocks. Thus there is no torque on the tilt plane, i.e., there is no torque on the smectic layer dipoles. Inside the grain boundaries, however, there are regions where the electric field is essentially along the tilt plane. On applying the field, these regions (with the width $l_{2}$ in Fig. 13) tend to expand as the free-energy density is minimized when the tilt plane is along the field. This expansion effectively "pushes" the dispirations out of the liquid crystal, and as the number of dispirations decreases for the same number of smectic blocks there is an increase of the helical pitch. On further increasing the field, the system reaches a point where the overall free energy becomes lower for the fingerprint texture than for the Grandjean texture and, hence, there is a transition to the fingerprint texture. This can be understood from the fact that in the fingerprint texture a larger part of the sample has the local tilt plane along the electric field than in the Grandjean texture. This phenomenon is analogous to the field-induced Grandjean to fingerprint transition in a cholesteric liquid crystal. Again the $x$ semiaxis of the local biaxial dielectric tensor of the anticlinic structure plays the role of the director in the cholesteric. In the fingerprint texture, the increasing electric field further unwinds the TGB helix as the local tilt plane is reoriented into the field direction. Finally, the TGB helix is squeezed out and we are left with a field-induced biaxial $\mathrm{SmC}_{a}^{*}$ structure.

\section{E. The tilt plane orientation in AFLCDs}

The tilt plane orientation in AFLC display applications has a significant impact on the electro-optic performance. In order to optimize the static dark state and to minimize the dynamic light leakage due to the pretransitional effect in a passive matrix drive, the AFLC should be horizontally surface stabilized and the tilt angle should preferably be $45^{\circ}$. The high speed of the linear electro-optic response $(\tau$ $\approx 10 \mu \mathrm{s}$ ) of the pretransitional effect suggests that it is due to the field-induced distortion (antiphase motion on the smectic cone in adjacent layers) of the antiferroelectric state. This effect is electro-optically active when the tilt plane is not parallel to the glass plates, e.g., when there is a helix present, when the surface stabilization is not complete, or when the surface-stabilized AFLC has undergone a Frederiks transition to a more or less vertical tilt plane state. Thus, in order to avoid the pretransitional effect, the AFLC should be horizontally surface stabilized up to the field strength at which the $\mathrm{AF} \rightarrow \mathrm{F}$ transition occurs. In other words, the Frederiks transition should be ruled out. We propose that this can be achieved by taking advantage of the fact that the Frederiks transition has a voltage threshold whereas the switching from the antiferroelectric to ferroelectric state (AF-F switching) has a field threshold [16]. By decreasing the cell thickness, the voltage required for the $\mathrm{AF} \rightarrow \mathrm{F}$ transition decreases while the Frederiks threshold voltage remains constant. Thus, there should be a cell thickness for which the Frederiks transition and the $\mathrm{AF} \rightarrow \mathrm{F}$ transition occurs at the same applied voltage. The binary mixture studied in Fig. 11 has an approximate threshold field for the AF $\rightarrow \mathrm{F}$ transition of $\mathbf{E}_{\mathrm{th}}=5.0 \mathrm{~V} / \mu \mathrm{m}$, while the onset of the Frederiks transition occurs at a field value $\mathbf{E}_{\mathrm{f}}=2.2 \mathrm{~V} / \mu \mathrm{m}$, indicating that the Frederiks transition should be ruled out at a cell thickness of less than about $1 \mu \mathrm{m}$. This aspect of the electro-optic processes determining the hysteresis shape is obviously very critical for applications and is now being studied in detail for different AFLC materials. There is still very little known about the requirements for an AFLC material to become surface stabilized in bookshelf cells. But it is clear that strongly planar anchoring combined with weakly polar anchoring $\left(\mathbf{P}_{s}\right.$ pointing into the surface has similar energy to $\mathbf{P}_{s}$ pointing out from the surface) promotes the HSS state. Furthermore, the cell thickness should be small, of the order of the helical pitch, in order for the helix free structure at the surface to penetrate the bulk of the cell.

\section{DISCUSSION AND CONCLUSIONS}

AFLC devices are generally considered to be working in a surface-stabilized bookshelf geometry, where all molecules are in the plane of the cell. But there has been no evidence for this to be true. Our analysis and experiments show that a large pretransitional effect is likely to be a result of the presence of a helix or incomplete surface stabilization. The helical structure presents a rotating tilt plane which accordingly is more or less perpendicular to the glass plates in about $50 \%$ of the cell area. Thus, the electric field couples to $\mathbf{P}_{s}$ of the individual smectic layers and distorts the antiferroelectric structure (linear electro-optic response), which in turn gives a net polarization in the local tilt plane. This induced polarization is reoriented into the field direction and unwinds the helix (quadratic electro-optic response). Following this 
analysis, it stands out as a major task to understand and be able to control the surface-stabilization mechanisms in AFLC cells.

We have shown that the tilt plane orientation can be probed by means of dielectric spectroscopy as all three relevant dielectric modes disappear in bookshelf cells when the cell becomes horizontally surface stabilized. These experiments were performed on orthoconic AFLCs in which we could optically verify the quality of the surface-stabilized state since it provides a perfectly dark state between crossed polarizers independent of the direction of the smectic layer normal. We could also study the Frederiks transition in surface-stabilized cells of a pitch compensated binary AFLC mixture by means of dielectric spectroscopy and electrooptic measurements. The dielectric spectra could be directly related to the electro-optic response, which strongly supports the origin of the dielectric modes in AFLCs discussed here.

One might argue that antitilt fluctuations (with electrocliniclike fluctuations in each smectic layer) could give an absorption in the recorded spectrum. Moreover, this mode should give the strongest contribution to the permittivity when the tilt plane is parallel to the glass plates. As no response, optical or dielectric, was detected in perfectly horizontally surface stabilized orthoconic AFLC cells, we could exclude antitilt fluctuations from being responsible for the modes observed.

The electric field effects in the $\mathrm{TGBC}_{a}$ phase can be understood by assuming that the local tilt plane inside a smectic block is perpendicular to the TGB helix axis, whereas it is parallel and perpendicular to this axis in a periodic fashion in the grain boundaries. The thickness of TGB smectic blocks is likely to be of the order of 20-100 nm, which means that the regions in the vicinity of the grain boundaries where the tilt plane is not perpendicular to the TGB helix constitute a relatively large part of the volume of the structure. In dielectric spectroscopy of the TGB phase, there are no drastic differences in the spectra of Grandjean and fingerprint texture cells. Low-frequency modes appear in both cases, indicating that virtually all directions of the tilt plane are represented in both textures. This gives further support to the proposed structure [20] of the $\mathrm{TGBC}_{a}$ phase in Fig. 13.

Finally, electro-optic effects in AFLCs below the switching to the ferroelectric states can be understood in terms of the tilt plane orientation. In many cases, e.g., the fieldinduced unwinding of the AFLC helix and the Frederiks transition in surface-stabilized cells, the $x$ axis of the local biaxial dielectric tensor plays the role of the director in the cases of unwinding of the cholesteric helix and the Frederiks transition in a planar aligned nematic.

\section{ACKNOWLEDGMENTS}

This work was supported by the Swedish Research Council, the TMR network ORCHIS, and the Swedish Defence Material Administration. The liquid crystalline samples were kindly provided by the groups of Professor Dabrowski (Warsaw) and Professor Heppke (Berlin).
[1] A. Fukuda, Y. Takanishi, T. Isozaki, K. Ishijawa, and H. Takezoe, J. Mater. Chem. 4, 997 (1994).

[2] K. D'havé, P. Rudquist, S. T. Lagerwall, H. Pauwels, W. Drzewinski, and R. Dabrowski, Appl. Phys. Lett. 76, 3528 (2000).

[3] K. D’havé, A. Dahlgren, P. Rudquist, J. P. F. Lagerwall, G. Andersson, M. Matuszczyk, S. T. Lagerwall, R. Dabrowski, and W. Drzewinski, Ferroelectrics 244, 115 (2000).

[4] J. W. Goodby, A. Petrenko, M. Hird, R. A. Lewis, J. Meier, and J. C. Jones, Chem. Commun. (Cambridge) 13, 1149 (2000); A. S. Petrenko, M. Hird, R. A. Lewis, J. G. Meier, J. C. Jones, and J. W. Goodby, J. Phys.: Condens. Matter 12, 8577 (2000).

[5] E. Gorecka, A. D. L. Chandani, H. Takezoe, and A. Fukuda, Jpn. J. Appl. Phys., Part 1 29, 131 (1990).

[6] L. A. Parry-Jones and S. J. Elston, Phys. Rev. E 63, 050701(R) (2001).

[7] L. A. Judge, E. E. Kriezis, and S. J. Elston, Mol. Cryst. Liq. Cryst. Sci. Technol., Sect. A 366, 661 (2001).

[8] A. Roy and N. V. Madhusudana, Eur. Phys. J. E. 1, 319 (2000).

[9] A. Roy and N. V. Madhusudana, Europhys. Lett. 41, 501 (1998)

[10] E. B. Priestly, P. J. Woitowicz, and P. Sheng, Introduction to Liquid Crystals (Plenum, New York, 1975).

[11] W107 is a four-component orthoconic AFLC mixture synthe- sized by the group of R. Dabrowski, Warsaw. For more information, see Ref. [3].

[12] H. Moritake, N. Shigeno, M. Ozaki, and K. Yoshino, Liq. Cryst. 14, 1283 (1993).

[13] P. Rudquist, D. Krüerke, S. T. Lagerwall, J. E. Maclennan, N. A. Clark, and D. M. Walba, Ferroelectrics 246, 927 (2000).

[14] K. Hiraoka, H. Takezoe, and A. Fukuda, Ferroelectrics 147, 113 (1993).

[15] T. Z. Qian and P. L. Taylor, Phys. Rev. E 60, 2978 (2000).

[16] B. Wen, S. Zhang, S. S. Keast, M. E. Neubert, P. L. Taylor, and P. L. C. Rosenblatt, Phys. Rev. E 62, 8152 (2000); S. Zhang, B. Wen, S. S. Keast, M. E. Neubert, P. L. Taylor, and P. L. C. Rosenblatt, Phys. Rev. Lett. 84, 4140 (2000).

[17] S. R. Renn and T. C. Lubensky, Phys. Rev. A 38, 2132 (1988).

[18] J. W. Goodby, M. A. Waugh, S. M. Stein, E. Chin, R. Pindak, and J. S. Patel, J. Am. Chem. Soc. 111, 8119 (1989); J. W. Goodby, M. A. Waugh, S. M. Stein, E. Chin, R. Pindak, and J. S. Patel, Nature (London) 337, 447 (1989).

[19] H. T. Nguyen, A. Bouchta, L. Navailles, P. Barois, N. Isaert, R. J. Tweig, A. Maaroufi, and C. Destrade, J. Phys. II 2, 1889 (1992).

[20] J. G. Meier, P. Rudquist, A. S. Petrenko, J. W. Goodby, S. T. Lagerwall, Liq. Cryst. 29, 179 (2002). 